\title{
Histopathological study of colo-ileal carcinoma
}

\author{
SUSUMU MATSUKUMA, KENJI OKADA, HIROAKI TAKEO and KIMIYA SATO
}

Department of Pathology, Japan Self Defense Forces Central Hospital, Tokyo 154-0001, Japan

Received September 19, 2011; Accepted December 13, 2011

DOI: $10.3892 / 01.2011 .538$

\begin{abstract}
Cases of colo-ileal carcinoma (CIC), defined as intestinal carcinoma involving the right-sided colon and the ileum, are rarely encountered. The aim of this study was to elucidate the clinicopathological characteristics, which have been poorly understood, in such cases. A total of $16 \mathrm{CICs}$ were examined histologically and immunohistochemically. Microsatellite instability-related histology was also evaluated according to previously published models, such as MsPath and PREDICT. CICs included 14 adenocarcinomas and 2 mucinous adenocarcinomas. The CICs showed focal or diffuse cytokeratin 20 expression and 7 CICs showed focal cytokeratin 7 co-expression. MsPath and PREDICT scores ranged from 1.6 to 6.6 (mean, 3.14) and from 1.6 to 7.8 (mean, 3.86), respectively. Three CICs showed loss of MLH1 immunoreactivity. Prominent neutrophilia and cancerous lymphangiosis in Peyer's patches (CLPP) were found in 8 cases $(50 \%)$ and in 3 cases (18.8\%), respectively. Neither variable was associated with parameters such as gender, tumor size or poor prognosis. However, the PREDICT score in prominently neutrophilic CICs was significantly higher than that in CICs with non-prominent neutrophilia $(\mathrm{P}=0.004)$. Patients with CLPP-positive CICs were significantly younger than those with CLPP-negative CICs $(\mathrm{P}=0.031)$. This study showed that almost all CICs originate from the right-sided colon with possible high levels of microsatellite instability. Prominent neutrophilia may be an additional histological indicator for microsatellite instability. Prognosis-independent CLPP occasionally occurs in younger patients with CICs.
\end{abstract}

\section{Introduction}

The ileum and the right-sided colon originate from the same embryologic midgut; however, in adults, the ileocecal valve demarcates the colon from the ileum $(1,2)$. Moreover, the occurrence of ileal carcinoma is rare, but right-sided colon carcinoma is not uncommon (3). However, cases of intestinal

Correspondence to: Dr Susumu Matsukuma, Department of Pathology, Japan Self Defense Forces Central Hospital, 1-2-24 Ikejiri, Setagaya-ku, Tokyo 154-0001, Japan

E-mail: skuma@cocoa.plala.or.jp

Key words: ileocecal cancer, microsatellite instability, neutrophilia, Peyer's patches, right-sided colon carcinoma carcinoma involving the terminal ileum and the right-sided colonhave rarely encountered. Additionally, the clinicopathological variables of such cases remain unclear. Right-sided colon carcinoma may show microsatellite instability (MSI) associated with defects in a DNA mismatch repair (MMR) system (3-7), but such a relationship in ileal carcinoma is unknown. In the terminal ileum adjacent to the colon, there is a specialized cluster of lymphoid follicles, or Peyer's patches, contributing to antigen sampling and pathogen entry $(1,2,8)$. However, the histopathological features of the carcinomatous involvement of Peyer's patches have been poorly understood. In this study, intestinal carcinoma involving the ileum end and the right-sided colon (cecum and/or ascending colon) was defined as 'colo-ileal carcinoma' (CIC), and an attempt was made to elucidate its clinicopathological characteristics.

\section{Materials and methods}

Materials. The surgical pathology reports of intestinal carcinoma in 53 patients who had undergone surgical resection of the ileum, cecum and ascending colon at the Department of Pathology, Japan Self Defense Forces Central Hospital (1985 to 2010) and at the Division of Surgical Pathology, Mishuku Hospital (1990 to 2010), Tokyo, Japan, were searched to identify cases of CIC. Cases of carcinoma originating in the vermiform appendix were excluded. A total of 16 CICs were retrieved and examined. Detailed clinical information including patients' personal history and family history was available in 11 cases from patient charts, none of which included history of inflammatory bowel disease.

Our study was approved by the ethics committees of both Japan Self Defense Forces Central hospital and Mishuku hospital. We obtained general informed consent for further study from patients who were included in this study.

Methods. All 16 CICs in their entirety were cut, formalin-fixed and paraffin-embedded in 4-11 blocks. Tumors were categorized using the World Health Organization classification of tumors of the colon and rectum (3). According to previously proposed models predicting a high level of MSI (MSI-H), i.e., MsPath (5) and PREDICT (6), tumor-infiltrating lymphocytes, Crohn's-like lymphocytic reaction, poorly differentiated or medullary components, peritumoral lymphocytic reaction, increased stromal plasma cells and mucinous adenocarcinoma components were assessed. The mucinous adenocarcinoma component was evaluated by two methods: i) for MsPath it was defined as at least $50 \%$ of the tumor area comprising mucinous 
features (5); and ii) for PREDICT it was scored dichotomously if CICs had mucinous features in more than $50 \%$ of the tumor area or if there was a focal mucinous area within a predominantly non-mucinous morphology (6). Representative sections were immunostained with antibodies against cytokeratin 7 (CK7; OV-TL 12/30, Dako, Glostrup, Denmark), cytokeratin 20 (CK20; Ks20.8, Dako), D2-40 (Dako), CD34 (QBEnd 10; Beckman Coulter, Marseille, France) and MLH1 (ES05; Leica Microsystems, Newcastle, UK). Statistical analysis was carried out using the Chi-square test, Fisher's exact test, Mann-Whitney U-test and log-rank test, as appropriate. A statistically significant difference was indicated when $\mathrm{P}<0.05$.

\section{Results}

Clinicopathological characteristics of patients and CIC. The main clinicopathological findings are shown in Table I. Patients with CICs included 7 males and 9 females ranging in age from 26 to 85 years (mean, 60.5). Four patients $(25.0 \%)$ were diagnosed before the age of 50 years, and four patients $(25.0 \%)$ were diagnosed between the ages of 50 and 59 years. Only two patients (Cases 14 and 16) had synchronous colorectal and gastric carcinomas; the mother of the youngest patient (Case 14, 26 years) had been diagnosed with colorectal carcinoma at the age of 50 years. Sessile serrated adenoma was found close to one CIC (Case 7). CICs ranged from 2.9 to $9.0 \mathrm{~cm}$ in maximal diameter (mean, $5.54 \mathrm{~cm}$ ). One CIC (Case 15) was restricted to the ileocecal mucosa, but the other CICs invaded the muscularis propria or subserosa and/or pericolic tissues. Two CICs (Cases 13 and 14) were mucinous adenocarcinoma involving $>50 \%$ of the tumor. The remaining 14 CICs showed features of tubular adenocarcinoma, and 7 (50\%) of the 14 CICs were accompanied by focal ( $<50 \%$ of tumor) mucinous adenocarcinoma components. Tumor-infiltrating lymphocytes, poorly differentiated components, Crohn's-like lymphocytic reaction, peritumoral lymphocytic reaction and increased stromal plasma cells were found in 6, 5, 2, 2 and 5 cases, respectively. The calculated MsPath and PREDICT scores ranged from 1.6 to 6.6 (mean, 3.14) and from 1.6 to 7.8 (mean, 3.86), respectively. The CICs exhibited focal or diffuse CK20 expression and 7 CICs showed focal CK7 co-expression. In the immunohistochemical examination of MHL1, we assessed a decreased MHL1 expression in CIC compared with non-neoplastic colonic mucosa in each case. However, in two cases, such assessment could not be performed, as carcinoma cells and non-neoplastic mucosa were poorly immunostained with MLH1. Only three (21.4\%) of the remaining 14 CICs showed a decreased expression of MHL1.

Neutrophils appear to correlate with MSI-H. In eight (50\%) of 16 CICs, prominent neutrophilic infiltration was found in cancer tubules and the surrounding stroma (Fig. 1), but such features were not prominent in the non-neoplastic mucosa in each case. Of the eight cases of prominently neutrophilic CIC and 8 cases of $\mathrm{CIC}$ with non-prominent neutrophilia no significant differences were found in patients' age $(\mathrm{P}=0.42)$, gender $(\mathrm{P}=1)$, tumor size $(\mathrm{P}=0.16)$, decreased MLH1 expression $(\mathrm{P}=0.9)$ or patient outcome $(\mathrm{P}=0.26)$. However, the MsPath and PREDICT scores in prominently neutrophilic CICs appeared to be higher than those in CICs with non-prominent neutro-

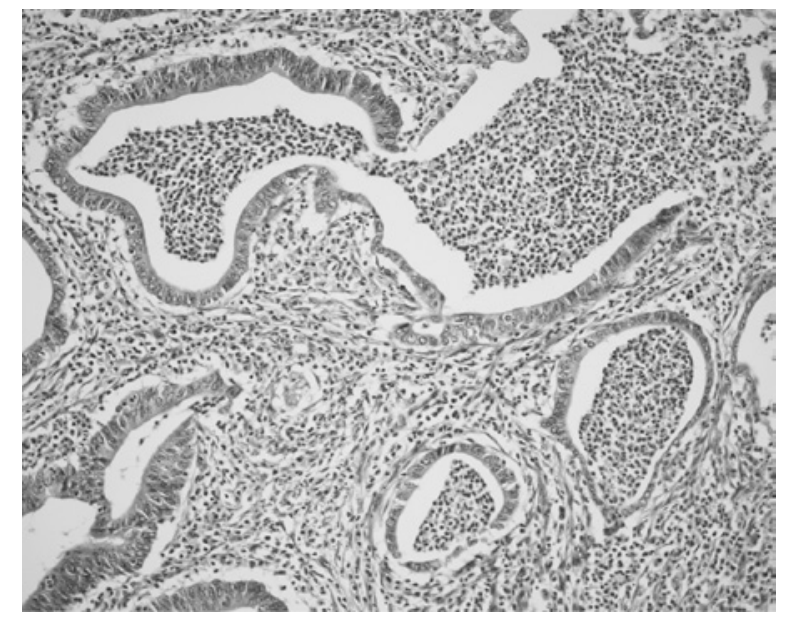

Figure 1. Numerous neutrophils involving both cancer tubules and the surrounding stroma (Case 5) (H\&E; original magnification, x200).

philia. Statistical analysis revealed a significant difference of the PREDICT score between 8 prominently neutrophilic CICs and the remaining 8 with non-prominent neutrophilia $(\mathrm{P}=0.004)$, although there was no significant difference in the total MsPath score between them $(\mathrm{P}=0.052)$.

Peyer's patches were involved in CIC. In three CICs (18.8\%), mucosal and/or submucosal dilated lymphatic spaces in Peyer's patches were prominently involved in carcinoma cell clusters (Fig. 2), suggesting cancerous lymphangiosis in Peyer's patches (CLPP). Endothelial cells lining CLPP spaces were almost always positive for D2-40, and occasionally showed CD34 coexpression. Three patients with CLPP-positive CICs ranged in age from 26 to 53 years (mean, 42.3), whereas 13 patients with CLPP-negative CICs ranged in age from 33 to 85 years (mean, 64.7); and there was a significant difference between the two groups $(\mathrm{P}=0.031)$. No significant associations were found between the presence of CLPP and clinicopathological characteristics such as gender $(\mathrm{P}=0.55)$, tumor size $(\mathrm{P}=0.31)$, the presence of lymph node metastasis $(\mathrm{P}=0.53)$, decreased MLH1 expression $(\mathrm{P}=0.5)$ or patient outcome $(\mathrm{P}=0.25)$. Additionally, no statistically significant differences were found in MsPath and PREDICT scores between them.

\section{Discussion}

In this study, all 16 CICs showed CK20 expression with or without CK7 co-expression, which did not show whether these CICs had originated in the right-sided colon or the ileum (9). However, histopathological features of CICs were consistent with colon adenocarcinoma, and distinct features indicating ileal origin were not identified. The incidence of ileal carcinoma is known to be significantly lower than that of colon carcinoma (3). Therefore, we consider that almost all of the invasive CICs had originated in the right-sided colonic mucosa and then invaded the ileum.

Slater et al noted that patients with right-sided colon carcinomas are older than those with left-sided colon carcinomas (10). However, other studies have described that right-sided colon origin is one of the major risk factors of 


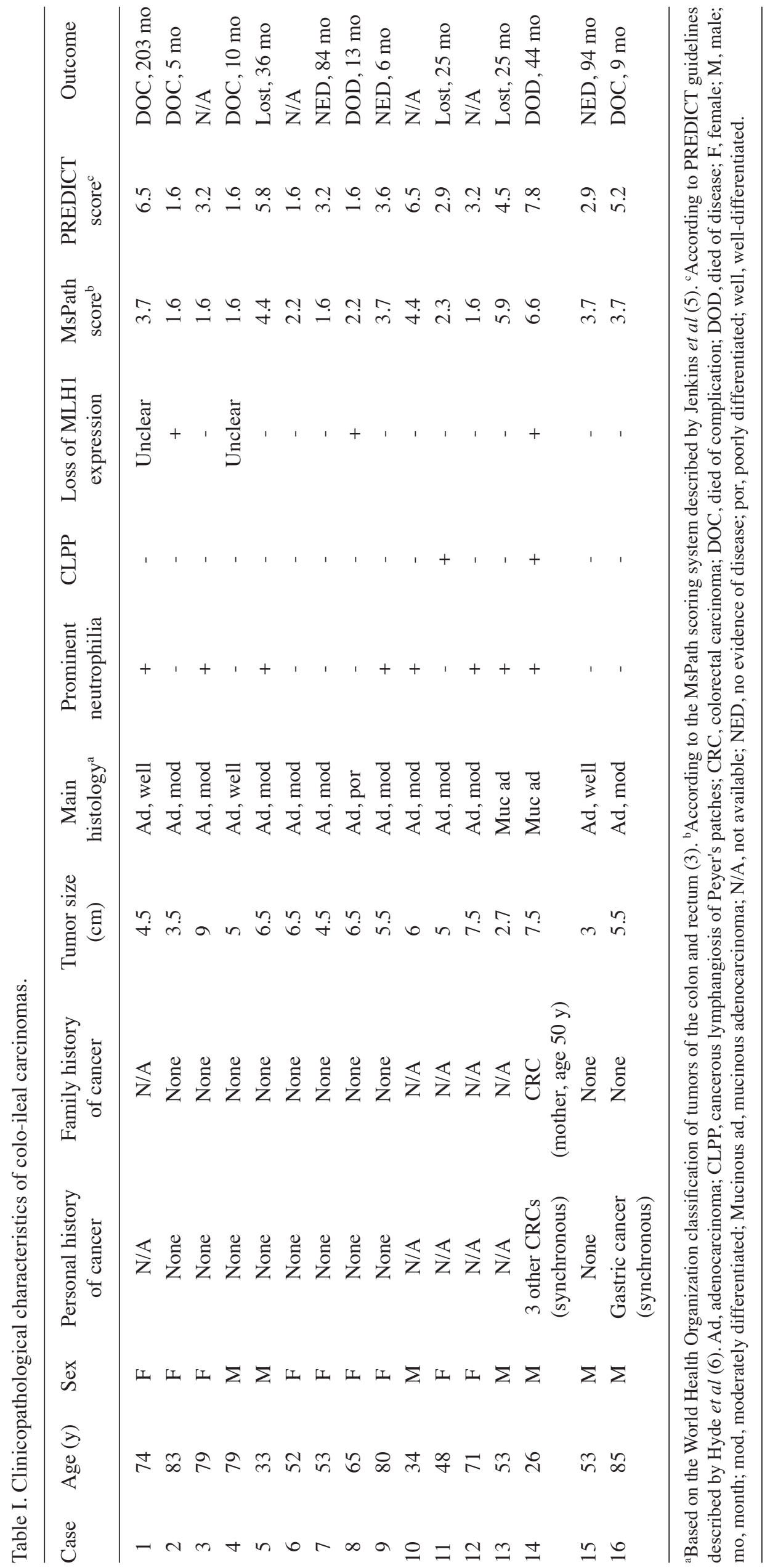


A

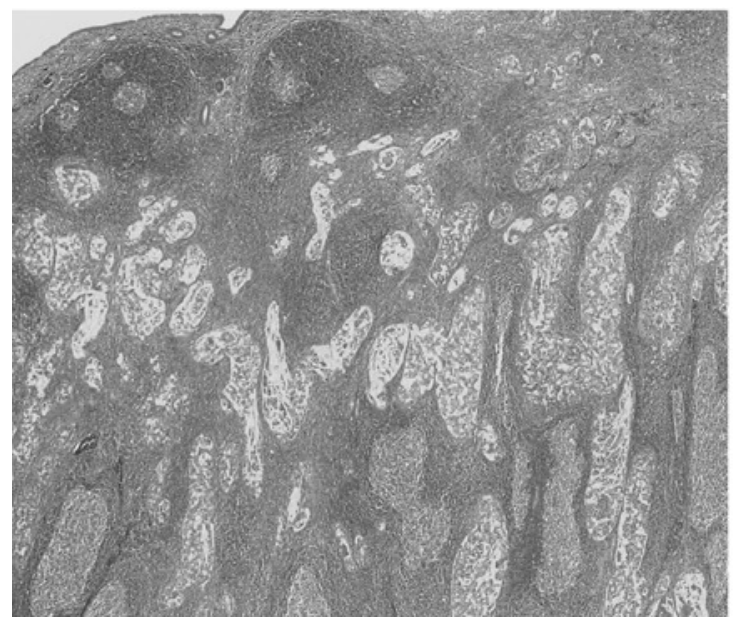

B

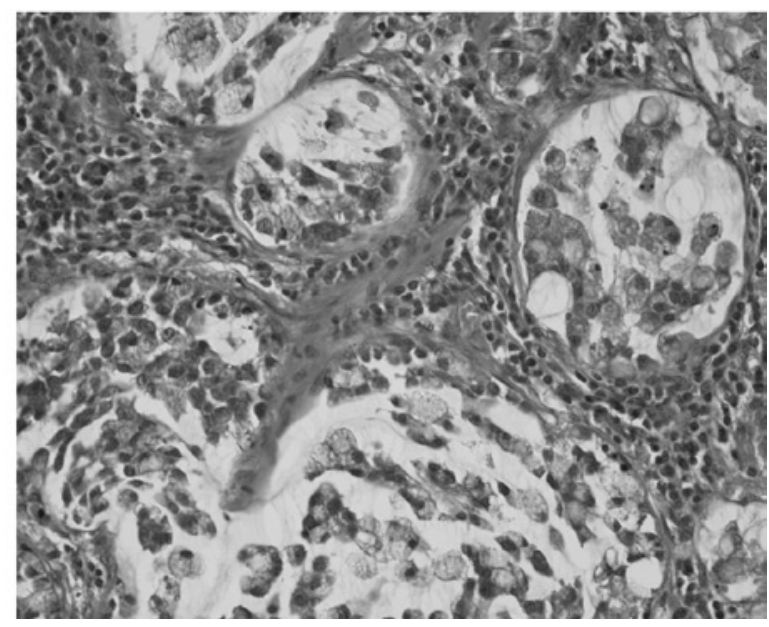

Figure 2. Lymphangiosis-like carcinomatous involvement of Peyer's patches of the terminal ileum (Case 14). (A) Low-power view showing numerous cancer nests involving Peyer's patch-associated lymphatic spaces (H\&E; original magnification, x40). (B) High-power view of lymphangiotic mucinous cancer cells. Additional immunostaining confirming D2-40-positive expression of the surrounding endothelial cells (H\&E; original magnification, $\mathrm{x} 400$ ).

MSI-related carcinoma, which is capable of developing in younger patients (3-7). In this study, the mean age of patients with CICs (60.5 years) was younger than that previously reported (71.3 years) for patients with right-sided colon carcinoma (10), which is attributable to the inclusion of younger MSI-related cases. Using MsPath (5) and PREDICT (6), findings of this study showed relatively high scores (mean MsPath score 3.14; mean PREDICT score 3.86), although there were no cases suggestive of Lynch syndrome $(3,4)$. However, in MsPath, only one finding of right-sided origin can add 1.6 to the score, and the previously recommended cut-off of 1.0 may not be specific for MSI-H $(5,6)$. Hyde et al reported that the specificity of MsPath without an age limit is $38.9 \%$ (6). Moreover, they also described that an arbitrary cut-off of 2.5 for PREDICT resulted in sensitivity of $96.9 \%$ and specificity of $76.6 \%$ in their validation cohort. In our series, this cut-off of 2.5 suggested that $12 \mathrm{CICs}$ are MSI-H, and the other group included 2 CICs with decreased MLH1 immunoexpression, suggestive of MSI-H tumors. Therefore, this study markedly suggested MSI-H status in 14 of 16 CICs (87.5\%). These 14 cases also included a sessile serrated adenoma-related case (Case 7) and the youngest patient (Case 14) who had multiple colorectal cancers and a mother with a history of colorectal cancer.

Previous studies have suggested that tumor-infiltrating lymphocytes, Crohn's-like lymphoid reactions, peritumoral lymphocytic reactions and stromal plasma cell infiltration are MSI-related reactions (3-7). Eosinophilia (11) and S-100 protein-positive dendritic cell infiltration (12) are also known to be good prognosis-associated inflammatory reactions in colorectal carcinoma. However, the clinicopathological significance of prominent neutrophilia in CIC or colorectal carcinoma remains unclear, although one study (13) reported no relationship between such features and MSI-H endometrial carcinoma. This study revealed prominent neutrophilia in 8 CICs $(8 / 16,50 \%)$, which were not associated with patients' age, gender, tumor size or prognosis. However, the PREDICT score in prominently neutrophilic CICs was significantly higher than that in CICs with non-prominent neutrophilia. The MsPath score in prominently neutrophilic CICs also appeared to be higher than that in CICs with non-prominent neutrophilia, but the difference showed borderline significance $(\mathrm{P}=0.052)$. These findings suggest that neutrophilia in CICs may be associated with MSI-H status.

In this study, CLPP was found in $18.8 \%$ of CICs. CLPP is an aggressive; however, no significant relationship was detected between the presence of CLPP and the presence of lymph node metastasis and a worse prognosis. CLPP was also not associated with gender, tumor size, decreased MLH1 expression or scores of MsPath and PREDICT. These findings suggest that CLPP is prognosis-independent and has nonspecific features. However, patients with CLPP-positive CICs were significantly younger than those with CLPP-negative CICs. In 2 of 3 patients with CICs showing CLPP, personal history was not available and there was the possibility of a history of inflammatory bowel disease. However, histopathological examination revealed no macroscopic or microscopic features indicating preexisting ulcerative colitis or Crohn's disease. Consequently, we consider that CLPP may be associated with age-related changes of Peyer's patches, which increase in size and number until puberty and regress steadily thereafter (2). Peyer's patches are composed of specialized cells or structures actively absorbing pathogens or antigens, and play a critical role in mucosal immunity $(1,2,8)$. Thus, lymphovascular flow and structures in the Peyer's patches may be somewhat different from those in other intestinal sites. These considerations may explain the occasional presence of non-specific CLPP in CICs and the frequent dual expression of D2-40 and CD34 in the endothelial cells lining the spaces around CLPP.

In conclusion, this study suggested that almost all CICs invade the ileum secondarily with a high frequency $(87.5 \%)$ of MIS-H status. Prominent neutrophilia may be an additional histological marker for MSI-H status. Prognosis-independent CLPP may occasionally occur in younger patients with CICs. 
However, this study included a limited number of examined cases, and more investigations are required to confirm our conclusions and findings.

\section{Acknowledgements}

The authors thank Drs A.O. Tadakazu, Michinori Murayama and Kimitoshi Inoue for kindly providing the study specimens; Haruyuki Nagasawa and Shin-ichi Katori for excellent technical assistance; and Daniel Mrozek for editing the manuscript.

\section{References}

1. Feldman M, Friedman LS and Brandt LJ (eds): Sleisenger and Fordtran's Gastrointestinal and Liver Disease. 8th edition. Saunders/Elsevier, Philadelphia, 2006.

2. Segal GH and Petras RE: Small Intestine. In: Histology for Pathologists, 2nd edition. Sternberg SS (ed). Lippincott-Raven Publishers, Philadelphia, pp495-518, 1997.

3. Hamilton SR and Aaltonen LA (eds): World Health Organization Classification of Tumours, Pathology and Genetics, Tumours of the Digestive System. IARC press, Lyon, 2000.

4. Umar A, Boland CR, Terdiman JP, et al: Revised Bethesda guidelines for hereditary nonpolyposis colorectal cancer (Lynch syndrome) and microsatellite instability. J Natl Cancer Inst 96: 261-268, 2004

5. Jenkins MA, Hayashi S, O'Shea AM, et al: Pathology features in Bethesda guidelines predict colorectal cancer microsatellite instability: a population-based study. Gastroenterology 133: $48-56,2007$.
6. Hyde A, Fontaine D, Stuckless S, et al: A histology-based model for predicting microsatellite instability in colorectal cancers. Am J Surg Pathol 34: 1820-1829, 2010.

7. Sinicrope F, Foster NR, Sargent DJ, et al: Model-based prediction of defective DNA mismatch repair using clinicopathological variables in sporadic colon cancer patients. Cancer 116: 1691-1698, 2010.

8. Lelouard H, Henri S, De Bovis B, et al: Pathogenic bacteria and dead cells are internalized by a unique subset of Peyer's patch dendritic cells that express lysozyme. Gastroenterology 138: 173-184, 2010.

9. Goldstein RS and Bosler DS: Immunohistochemistry of the gastrointestinal tract, pancreas, bile ducts, gallbladder and liver. In: Diagnostic Immunohistochemistry. 2nd edition. Dabbs DJ (ed). Churchill Livingston/Elsevier, Philadelphia, pp442-508, 2006.

10. Slater G, Papatestas AE, Tartter PI, Mulvihill M and Aufses AH: Age distribution of right- and left-sided colorectal cancers. Am J Gastroenterol 77: 63-66, 1982.

11. Pretlow TP, Keith EF, Cryar AK, et al: Eosinophil infiltration of human colonic carcinomas as a prognostic indicator. Cancer Res 43: 2997-3000, 1983.

12. Ambe K, Mori M and Enjoji M: S-100 protein-positive dendritic cells in colorectal adenocarcinomas. Distribution and relation to the clinical prognosis. Cancer 63: 496-503, 1989.

13. Shia J, Black D, Hummer J, Boyd J and Soslow RA: Routinely assessed morphological features correlate with microsatellite instability status in endometrial cancer. Hum Pathol 39: 116-125, 2008. 